\title{
Ultrasound-guided bilateral modified thoracolumbar interfascial plane or infiltration block for analgesia after lumbar spinal surgery: a prospective randomized open blinded end-point study
}

Hironobu Ueshima. Hiroshi Otake

\author{
Department of Anesthesiology, Showa University Hospital, Tokyo, Japan
}

\section{【Background and Aims】}

Bilateral modified thoracolumbar interfascial plane (TLIP) block provides effective analgesia for spinal surgery. Although an ultrasound machine is needed to provide effective modified TLIP block, is not needed for an infiltration block. We examined patients undergoing lumbar spinal surgery for comparing analgesic efficacy between bilateral modified TLIP and the infiltration blocks.

\section{【Methods】}

Sixty adult patients scheduled for lumbar spinal surgery were randomly allocated to receive bilateral modified TLIP block (T group) or infiltration block (I group) under general anaesthesia. The primary outcome was cumulative fentanyl administered for rescue analgesia, and secondary outcomes were the number of demands for rescue analgesia and successfully delivered rescue analgesia; pain scores on the visual analogue

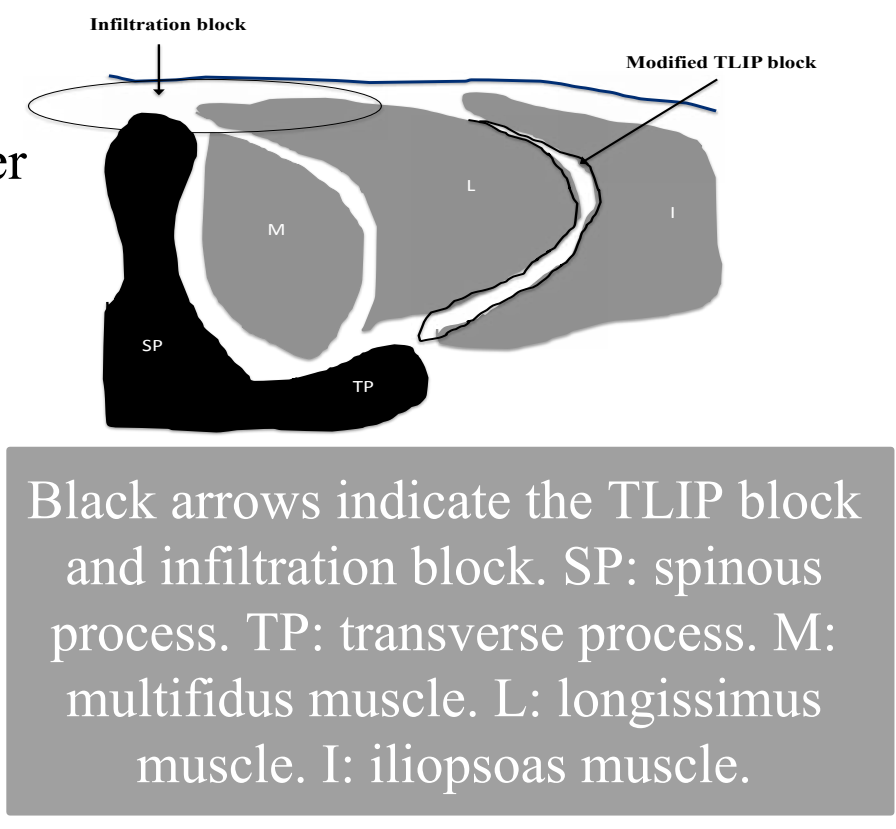
scale (VAS) at rest and during mobilisation; and extent of sensory level block during the first 48 hours after surgery.

【Results】

-Flow chart

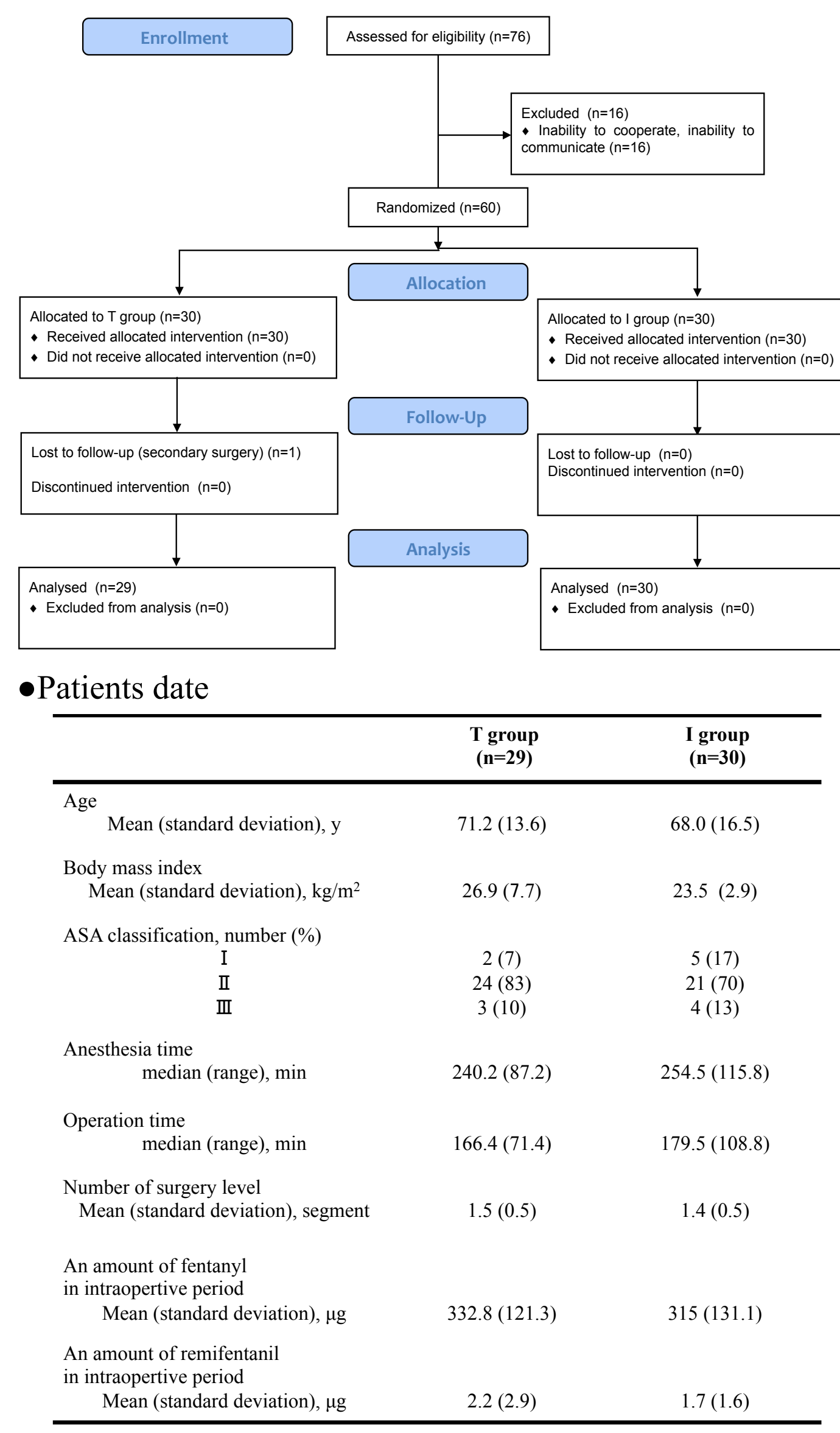

【Results】

-(A) Cumulative fentanyl volume in the $T$ group and I group within the first 24 and 48 hours after surgery. (B) Number of demands for rescue analgesia $(L)$ and successfully delivered rescue analgesia $(R)$ in the $T$ group and I group within the first 24 and 48 hours after surgery. Data are expressed as mean (standard deviation) $(\mu \mathrm{g})$.

(A)

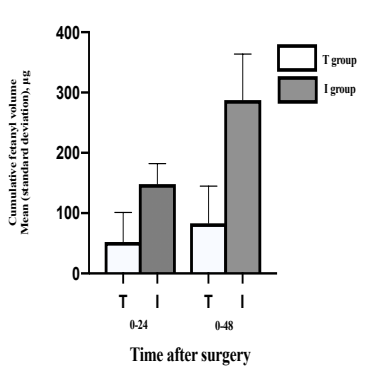

(B)
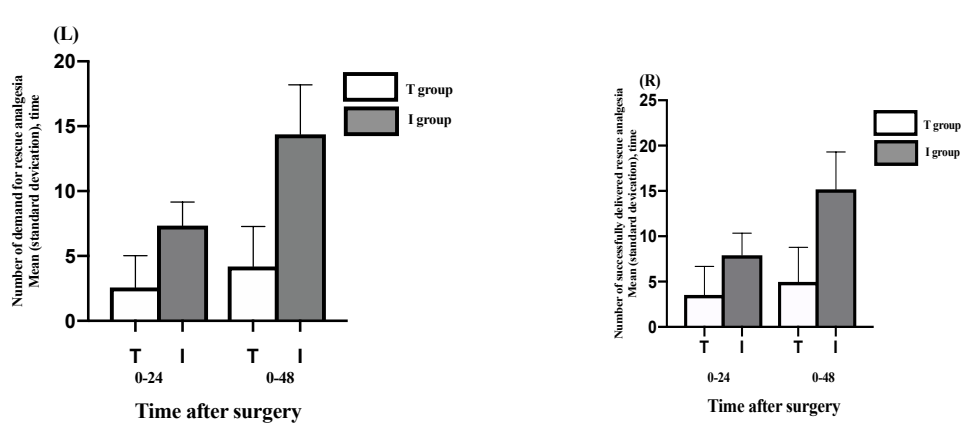

-VAS scores (since 2 hours postoperatively at rest and 6 hours postoperatively during mobilization) were lower in the T group than in the I group $(\mathrm{P}<0.001)$.

- Level of sensory block as determined by cold test.

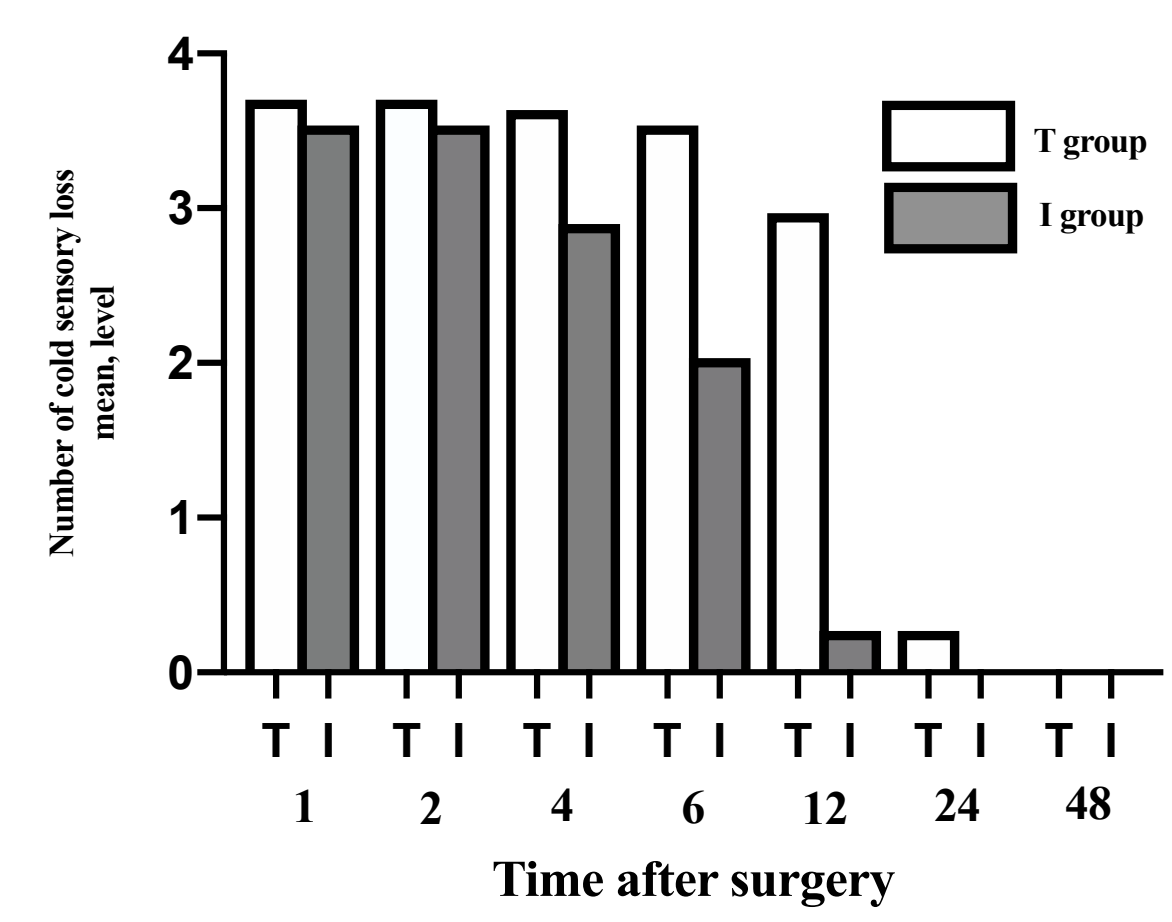

【Discussion】

Our results show that, in patients undergoing lumbar spinal surgery, the modified TLIP block can provide more effective and longer analgesia in the perioperative period compared to the infiltration block under general anesthesia. Considering the extent and duration of loss of cold sensation observed in this study, it can be concluded that the local anesthetic, which is injected into the interfascial plane in TLIP block can spread and block a wider range of spinal nerves compared to the infiltration block. Although the local anesthetic in the infiltration block, which is injected at the subcutaneous tissue and into the erector spinae muscle of the incised site, can block the cutaneous branch of the posterior branch of the spinal nerve, only this nerve is blocked around the injection site and it cannot block the wider spinal nerves. In addition, the local anesthetic injected at the interfascial plane stays longer in this plane.

【Conclusions:】

Compared with infiltration block, bilateral modified TLIP block may provide effective analgesia for patients undergoing lumbar spinal surgery under general anaesthesia. 